\title{
Some Identities Involving the Derivative of the First Kind Chebyshev Polynomials
}

\author{
Tingting Wang ${ }^{1}$ and Han Zhang ${ }^{2}$ \\ ${ }^{1}$ College of Science, Northwest A\&F University, Yangling, Shaanxi 712100, China \\ ${ }^{2}$ School of Mathematics, Northwest University, Xian, Shaanxi 710127, China \\ Correspondence should be addressed to Han Zhang; micohanzhang@hotmail.com
}

Received 10 May 2015; Accepted 11 June 2015

Academic Editor: Filippo Ubertini

Copyright (c) 2015 T. Wang and H. Zhang. This is an open access article distributed under the Creative Commons Attribution License, which permits unrestricted use, distribution, and reproduction in any medium, provided the original work is properly cited.

We use the combinatorial method and algebraic manipulations to obtain several interesting identities involving the power sums of the derivative of the first kind Chebyshev polynomials. This solved an open problem proposed by Li (2015).

\section{Introduction}

For any integer $n \geq 0$, the famous Chebyshev polynomials of the first and second kind $T_{n}(x)$ and $U_{n}(x)$ are defined as follows:

$$
\begin{aligned}
& T_{n}(x)=\frac{n}{2} \sum_{k=0}^{[n / 2]}(-1)^{k} \frac{(n-k-1) !}{k !(n-2 k) !}(2 x)^{n-2 k}, \\
& U_{n}(x)=\sum_{k=0}^{[n / 2]}(-1)^{k} \frac{(n-k) !}{k !(n-2 k) !}(2 x)^{n-2 k},
\end{aligned}
$$

where $[n / 2]$ denotes the greatest integer $\leq n / 2$.

It is clear that $T_{n}(x)$ and $U_{n}(x)$ are the second-order linear recurrence polynomials; they satisfy the recurrence formulae:

$$
\begin{aligned}
& T_{0}(x)=1, T_{1}(x)=x, \text { and } T_{n+1}(x)=2 x T_{n}(x)-T_{n-1}(x) \\
& \text { for all } n \geq 1, \\
& U_{0}(x)=1, U_{1}(x)=2 x, \text { and } U_{n+1}(x)=2 x U_{n}(x)- \\
& U_{n-1}(x) \text { for all } n \geq 1 .
\end{aligned}
$$

The general term formulae of $T_{n}(x)$ and $U_{n}(x)$ are

$$
\begin{aligned}
& T_{n}(x)=\frac{1}{2}\left[\left(x+\sqrt{x^{2}-1}\right)^{n}+\left(x-\sqrt{x^{2}-1}\right)^{n}\right], \\
& U_{n}(x) \\
& =\frac{1}{2 \sqrt{x^{2}-1}}\left[\left(x+\sqrt{x^{2}-1}\right)^{n+1}-\left(x-\sqrt{x^{2}-1}\right)^{n+1}\right] .
\end{aligned}
$$

Both $T_{n}(x)$ and $U_{n}(x)$ are orthogonal polynomials. That is,

$$
\begin{aligned}
& \int_{-1}^{1} \frac{T_{m}(x) T_{n}(x)}{\sqrt{1-x^{2}}} d x= \begin{cases}0, & \text { if } m \neq n ; \\
\frac{\pi}{2}, & \text { if } m=n>0 ; \\
\pi, & \text { if } m=n=0,\end{cases} \\
& \int_{-1}^{1} \sqrt{1-x^{2}} U_{m}(x) U_{n}(x) d x= \begin{cases}0, & \text { if } m \neq n ; \\
\frac{\pi}{2}, & \text { if } m=n .\end{cases}
\end{aligned}
$$

About the other various properties of the Chebyshev polynomials, some authors had studied them and obtained many interesting conclusions. For example, Ma and Zhang [1], Wang and Han [2], Cesarano [3], and Lee and Wong [4] proved a series of identities involving Chebyshev polynomials. Bhrawy and others (see [5-10]) obtained many important applications of the Chebyshev polynomials.

Very recently, Li [11] proved some identities involving power sums of $T_{n}(x)$ and $U_{n}(x)$. That is, for any positive integers $h$ and $n$, one has the identities:

(a)

$$
\sum_{m=0}^{h} T_{2 m+1}^{2 n+1}(x)=\frac{1}{2^{2 n+1}} \sum_{k=0}^{n}\left(\begin{array}{c}
2 n+1 \\
n-k
\end{array}\right) \frac{U_{2(2 k+1)(h+1)-1}(x)}{U_{2 k}(x)},
$$


(b)

$$
\begin{aligned}
& \sum_{m=0}^{h} U_{2 m}^{2 n+1}(x) \\
& \quad=\frac{1}{4^{n}\left(x^{2}-1\right)^{n}} \sum_{k=0}^{n}\left(\begin{array}{c}
2 n+1 \\
n-k
\end{array}\right)(-1)^{n-k} \frac{U_{(2 k+1)(h+1)-1}^{2}(x)}{U_{2 k}(x)},
\end{aligned}
$$

(c)

$$
\begin{aligned}
& \sum_{m=1}^{h} T_{2 m}^{2 n+1}(x) \\
& \quad=\frac{1}{2^{2 n+1}} \sum_{k=0}^{n}\left(\begin{array}{c}
2 n+1 \\
n-k
\end{array}\right) \frac{U_{(2 k+1)(2 h+1)-1}(x)-U_{2 k}(x)}{U_{2 k}(x)},
\end{aligned}
$$

(d)

$$
\begin{aligned}
& \sum_{m=1}^{h} U_{2 m-1}^{2 n+1}(x)=\frac{1}{2^{2 n+1}\left(x^{2}-1\right)^{n+1}} \\
& \quad \sum_{k=0}^{n}\left(\begin{array}{c}
2 n+1 \\
n-k
\end{array}\right)(-1)^{n-k} \frac{T_{(2 k+1)(2 h+1)}(x)-T_{2 k+1}(x)}{U_{2 k}(x)} .
\end{aligned}
$$

As for some applications of these results, Xiaoxue $\mathrm{Li}$ obtained some divisibility properties involving Chebyshev polynomials. At the same time, she also proposed the following open problem.

Does there exist an exact expression for the derivative or integral of the Chebyshev polynomials of the first kind in terms of the Chebyshev polynomials of the first kind (and vice versa)? That is to say, does there exist an exact expression for the summations

$$
\begin{gathered}
\sum_{m=1}^{h}\left(T_{m}^{\prime}(x)\right)^{n} \\
\text { or } \sum_{m=1}^{h}\left(\int_{0}^{x} T_{m}(y) d y\right)^{n}
\end{gathered}
$$

in terms of the Chebyshev polynomials of the first kind?

Does there exist an exact expression for $\sum_{m=1}^{h} T_{m}^{n}(x)$ in terms of $T_{m}^{\prime}(x)$ or $\int_{0}^{x} T_{m}(y) d y$ where $T_{m}^{\prime}(x)$ denotes the derivative of $T_{m}(x)$ with respect to $x$ ?

In this paper, as a note of [11], we give some identities involving the derivative of the first kind Chebyshev polynomials. That is, we will prove the following.
Theorem 1. For any integers $h \geq 1$ and $n \geq 0$, one has the identity

$$
\begin{aligned}
\sum_{m=1}^{h}\left(T_{2 m}^{\prime}(x)\right)^{2 n+1}=\sum_{i=1}^{n+1} \sum_{k=0}^{n}\left(\begin{array}{c}
2 n+1 \\
n-k
\end{array}\right)(-1)^{n-k} & \cdot \frac{R(i, h) U_{2 i(2 k+1)-1}(x)+S(i, h) U_{(2 i-2)(2 k+1)-1}(x)}{4^{i}\left(x^{2}-1\right)^{n+i} U_{2 k}^{2 i}(x)} \\
+ & \sum_{i=1}^{n+1} \sum_{k=0}^{n}\left(\begin{array}{c}
2 n+1 \\
n-k
\end{array}\right)(-1)^{n-k} \\
& \cdot \frac{H(i, h) U_{(2 i+2 h)(2 k+1)-1}(x)+K(i, h) U_{(2 i-2+2 h)(2 k+1)-1}(x)}{4^{i}\left(x^{2}-1\right)^{n+i} U_{2 k}^{2 i}(x)}
\end{aligned}
$$

where $R(i, h), S(i, h), H(i, h)$, and $K(i, h)$ are computable constants.

Theorem 2. For any positive integers $h$ and $n$, one has the identity

$$
\begin{aligned}
\sum_{m=1}^{h}\left(T_{m}^{\prime}(x)\right)^{2 n}=\frac{(-1)^{n}}{4^{n}\left(x^{2}-1\right)^{n}} \cdot \frac{(2 n) !}{(n !)^{2}} \cdot\left(\sum_{m=1}^{h} m^{2 n}\right) \\
+\sum_{i=1}^{n+1} \sum_{k=1}^{n}\left(\begin{array}{c}
2 n \\
n-k
\end{array}\right)(-1)^{n-k} \\
+\frac{r(i, h) T_{2 k i}(x)+s(i, h) T_{2 k(i-1)}(x)}{4^{n+i}\left(x^{2}-1\right)^{n+i} U_{k-1}^{2 i}(x)} \\
+\sum_{i=1}^{n+1} \sum_{k=1}^{n}\left(\begin{array}{c}
2 n \\
n-k
\end{array}\right)(-1)^{n-k} \\
\cdot \frac{p(i, h) T_{2 k(h+i)}(x)+q(i, h) T_{2 k(h+i-1)}(x)}{4^{n+i}\left(x^{2}-1\right)^{n+i} U_{k-1}^{2 i}(x)},
\end{aligned}
$$

where $r(i, h), s(i, h), p(i, h)$, and $q(i, h)$ are computable constants.

For some special $n$, from Theorem 1 with $n=0$ and Theorem 2 with $n=1$, we can also deduce the following two corollaries.

Corollary 3. For any positive integer h, one has the identity

$$
\sum_{m=1}^{h} T_{2 m}^{\prime}(x)=\frac{h U_{2 h+1}(x)-(h+1) U_{2 h-1}(x)}{2\left(x^{2}-1\right)} .
$$

Corollary 4. For any positive integer $h$, one has the identity

$$
\begin{aligned}
\sum_{m=1}^{h} & \left(T_{m}^{\prime}(x)\right)^{2} \\
& =-\frac{h(h+1)(2 h+1)}{12\left(x^{2}-1\right)}
\end{aligned}
$$




$$
\begin{aligned}
& +\frac{\left(h^{2}-2\right) T_{2 h+2}(x)-(h+1)^{2} T_{2 h}(x)}{8\left(x^{2}-1\right)^{2}} \\
& +\frac{T_{2 h+4}(x)-T_{2 h+2}(x)}{16\left(x^{2}-1\right)^{3}} .
\end{aligned}
$$

\section{Two Simple Lemmas}

In this section, we will give two simple lemmas, which are necessary in the proofs of our theorems. First we express $\left(T_{m}^{\prime}(x)\right)^{2 n}$ and $\left(T_{m}^{\prime}(x)\right)^{2 n+1}$ in terms of $T_{k}(x)$ and $U_{k}(x)$. That is, we have the following.

Lemma 1. For any positive integers $m$ and $n$, one has the identities

$$
\begin{aligned}
& \left(T_{m}^{\prime}(x)\right)^{2 n}=\frac{1}{4^{n}\left(x^{2}-1\right)^{n}}\left[(-1)^{n} \frac{(2 n) !}{(n !)^{2}} m^{2 n}\right. \\
& \left.+2 \sum_{k=1}^{n}\left(\begin{array}{c}
2 n \\
n-k
\end{array}\right)(-1)^{n-k} m^{2 n} T_{2 k m}(x)\right], \\
& \left(T_{m}^{\prime}(x)\right)^{2 n+1}=\frac{1}{4^{n}\left(x^{2}-1\right)^{n}} \\
& \quad \cdot \sum_{k=0}^{n}\left(\begin{array}{c}
2 n+1 \\
n-k
\end{array}\right)(-1)^{n-k} m^{2 n+1} U_{m(2 k+1)-1}(x) .
\end{aligned}
$$

Proof. For any positive integer $n$ and real number $x \neq 0$ and 1 , by using the familiar binomial expansion we have

$$
\begin{aligned}
& \left(y-\frac{1}{y}\right)^{2 n} \\
& =(-1)^{n} \frac{(2 n) !}{(n !)^{2}}+\sum_{k=1}^{n}\left(\begin{array}{c}
2 n \\
n-k
\end{array}\right)(-1)^{n-k}\left(y^{2 k}+\frac{1}{y^{2 k}}\right), \\
& \left(y-\frac{1}{y}\right)^{2 n+1} \\
& =\sum_{k=0}^{n}\left(\begin{array}{c}
2 n+1 \\
n-k
\end{array}\right)(-1)^{n-k}\left(y^{2 k+1}-\frac{1}{y^{2 k+1}}\right) .
\end{aligned}
$$

Now we take $y=\left(x+\sqrt{x^{2}-1}\right)^{m}$ in (14); note that $1 / y=$ $\left(x-\sqrt{x^{2}-1}\right)^{m}$; from the definition of $T_{n}(x)$ and $U_{n}(x)$ we have the identities

$$
\begin{aligned}
& U_{m-1}^{2 n}(x)=\frac{1}{4^{n}\left(x^{2}-1\right)^{n}}\left[(-1)^{n} \frac{(2 n) !}{(n !)^{2}}\right. \\
& \left.+2 \sum_{k=1}^{n}\left(\begin{array}{c}
2 n \\
n-k
\end{array}\right)(-1)^{n-k} T_{2 k m}(x)\right],
\end{aligned}
$$

$$
\begin{aligned}
& U_{m-1}^{2 n+1}(x)=\frac{1}{4^{n}\left(x^{2}-1\right)^{n}} \\
& \quad \sum_{k=0}^{n}\left(\begin{array}{c}
2 n+1 \\
n-k
\end{array}\right)(-1)^{n-k} U_{m(2 k+1)-1}(x) .
\end{aligned}
$$

Note that $T_{n}^{\prime}(x)=n U_{n-1}(x)$; from (15) we may immediately deduce

$$
\begin{aligned}
& \left(T_{m}^{\prime}(x)\right)^{2 n}=\frac{1}{4^{n}\left(x^{2}-1\right)^{n}}\left[(-1)^{n} \frac{(2 n) !}{(n !)^{2}} m^{2 n}\right. \\
& \left.+2 \sum_{k=1}^{n}\left(\begin{array}{c}
2 n \\
n-k
\end{array}\right)(-1)^{n-k} m^{2 n} T_{2 k m}(x)\right], \\
& \left(T_{m}^{\prime}(x)\right)^{2 n+1}=\frac{1}{4^{n}\left(x^{2}-1\right)^{n}} \\
& \cdot \sum_{k=0}^{n}\left(\begin{array}{c}
2 n+1 \\
n-k
\end{array}\right)(-1)^{n-k} m^{2 n+1} U_{m(2 k+1)-1}(x) .
\end{aligned}
$$

This proves Lemma 1.

Lemma 2. Let $h$ and $k$ be two positive integers. Then for any $x \neq 0$ and 1 , we have the recurrence formula:

(I)

$$
\begin{aligned}
\sum_{m=1}^{h} m^{k} x^{m}= & \frac{x}{1-x}+\frac{x}{1-x} \sum_{i=1}^{k-1}\left(\begin{array}{l}
k \\
i
\end{array}\right) \sum_{m=1}^{h} m^{i} x^{m} \\
& -\frac{(h+1)^{k} x^{h+1}}{1-x} .
\end{aligned}
$$

There exist some computable constants $C(r, s)$ and $D(r, s)$ such that

(II)

$$
\begin{aligned}
\sum_{m=1}^{h} m^{k} x^{m}= & \sum_{i=1}^{k+1} C(i, h) \cdot \frac{x^{i}}{(1-x)^{i}} \\
& +\sum_{i=1}^{k+1} D(i, h) \cdot \frac{x^{h+i}}{(1-x)^{i}} .
\end{aligned}
$$

In particular for $k=1$ and 2 , we have the identities:

(A)

$$
\sum_{m=1}^{h} m x^{m}=\frac{x-(h+1) x^{h+1}+h x^{h+2}}{(1-x)^{2}} ;
$$

(B)

$$
\begin{aligned}
\sum_{m=1}^{h} m^{2} x^{m}= & \frac{x+x^{2}}{(1-x)^{3}}-\frac{(h+1)^{2} x^{h+1}}{1-x}-\frac{(2 h+3) x^{h+2}}{(1-x)^{2}} \\
& -\frac{2 x^{h+3}}{(1-x)^{3}} .
\end{aligned}
$$


Proof. For any positive integer $k$, let $f(x, k, h)=\sum_{m=1}^{h} m^{k} x^{m}$; it is clear that

$$
\begin{aligned}
(1-x) f(x, k, h)= & \sum_{m=1}^{h} m^{k} x^{m}-\sum_{m=1}^{h} m^{k} x^{m+1} \\
= & \sum_{m=1}^{h} m^{k} x^{m}-\sum_{m=1}^{h-1} m^{k} x^{m+1}-h^{k} x^{h+1} \\
= & x+\sum_{m=1}^{h-1}(m+1)^{k} x^{m+1} \\
& -\sum_{m=1}^{h-1} m^{k} x^{m+1}-h^{k} x^{h+1} \\
= & x+\sum_{m=1}^{h-1}\left((m+1)^{k}-m^{k}\right) x^{m+1} \\
& -h^{k} x^{h+1} .
\end{aligned}
$$

Note that the binomial expansion is as follows:

$$
(m+1)^{k}-m^{k}=\sum_{i=0}^{k-1}\left(\begin{array}{l}
k \\
i
\end{array}\right) m^{i},
$$

and from (21) we may immediately deduce the identity:

$$
\begin{aligned}
& f(x, k, h)=\sum_{m=1}^{h} m^{k} x^{m} \\
& =\frac{x}{1-x}+\frac{x}{1-x} \sum_{i=0}^{k-1}\left(\begin{array}{l}
k \\
i
\end{array}\right) \sum_{m=1}^{h-1} m^{i} x^{m}-\frac{h^{k} x^{h+1}}{1-x} \\
& =\frac{x}{1-x}+\frac{x}{1-x} \sum_{i=0}^{k-1}\left(\begin{array}{l}
k \\
i
\end{array}\right) \sum_{m=1}^{h} m^{i} x^{m} \\
& -\frac{x}{1-x} \sum_{i=0}^{k-1}\left(\begin{array}{l}
k \\
i
\end{array}\right) h^{i} x^{h}-\frac{h^{k} x^{h+1}}{1-x} \\
& =\frac{x}{1-x}+\frac{x}{1-x} \sum_{i=0}^{k-1}\left(\begin{array}{l}
k \\
i
\end{array}\right) \sum_{m=1}^{h} m^{i} x^{m} \\
& -\frac{x^{h+1}}{1-x}\left((h+1)^{k}-h^{k}\right)-\frac{h^{k} x^{h+1}}{1-x} \\
& =\frac{x}{1-x}+\frac{x}{1-x} \sum_{i=0}^{k-1}\left(\begin{array}{l}
k \\
i
\end{array}\right) \sum_{m=1}^{h} m^{i} x^{m} \\
& -\frac{(h+1)^{k} x^{h+1}}{1-x} \\
& =\frac{x}{1-x}+\frac{x}{1-x} \sum_{i=0}^{k-1}\left(\begin{array}{l}
k \\
i
\end{array}\right) f(x, i, h) \\
& -\frac{(h+1)^{k} x^{h+1}}{1-x} \text {. }
\end{aligned}
$$

This proves the recurrence formula (I).
It is easy to prove (II) by recurrence formula (I) and the complete mathematical induction.

From the recurrence formula (I) and noting that the identity

$$
\sum_{m=1}^{h} x^{m}=\frac{x-x^{h+1}}{1-x}
$$

we can also deduce

$$
\begin{aligned}
\sum_{m=1}^{h} m x^{m} & =\frac{x}{1-x}+\frac{x}{1-x} \frac{x-x^{h+1}}{1-x}-\frac{(h+1) x^{h+1}}{1-x} \\
& =\frac{x-(h+1) x^{h+1}+h x^{h+2}}{(1-x)^{2}} .
\end{aligned}
$$

This proves formula (A).

Identity (B) follows from (24), (A), and the recurrence formula (I) with $k=2$. This proves Lemma 2 .

Some Note. The first part of Lemma 2 obtained an interesting recurrence formula for the computation of the summation $\sum_{m=1}^{h} m^{k} x^{m}$. But if positive integer $k$ is large enough, then the computation of the recurrence formula is more complex, and so we have not given the exact constants $C(i, h)$ and $D(i, h)$ in formula (II).

\section{Proofs of the Theorems}

In this section, we will complete the proofs of our theorems. First we prove Theorem 1. Let $\alpha=x+\sqrt{x^{2}-1}, \beta=$ $x-\sqrt{x^{2}-1}$; then note that $\alpha \cdot \beta=1,\left(1-\alpha^{2(2 k+1)}\right)=$ $-\alpha^{2 k+1}\left(\alpha^{2 k+1}-\beta^{2 k+1}\right)$; from the definition of $U_{n}(x)$ and the second formula of Lemma 1 we have

$$
\begin{aligned}
& \sum_{m=1}^{h}\left(T_{2 m}^{\prime}(x)\right)^{2 n+1}=\frac{1}{4^{n}\left(x^{2}-1\right)^{n}} \sum_{k=0}^{n}\left(\begin{array}{c}
2 n+1 \\
n-k
\end{array}\right)(-1)^{n-k} \\
& \cdot \sum_{m=1}^{h}(2 m)^{2 n+1} U_{2 m(2 k+1)-1}(x)=\frac{1}{\left(x^{2}-1\right)^{n+1 / 2}} \\
& \cdot \sum_{k=0}^{n}\left(\begin{array}{c}
2 n+1 \\
n-k
\end{array}\right)(-1)^{n-k} \\
& \cdot \sum_{m=1}^{h}\left(m^{2 n+1} \alpha^{2 m(2 k+1)}-m^{2 n+1} \beta^{2 m(2 k+1)}\right) .
\end{aligned}
$$

Applying formula (II) of Lemma 2 we have

$$
\begin{gathered}
\sum_{m=1}^{h}\left(m^{2 n+1} \alpha^{2 m(2 k+1)}-m^{2 n+1} \beta^{2 m(2 k+1)}\right)=\sum_{i=1}^{2 n+2} C(i, h) \\
\cdot \frac{\alpha^{2 i(2 k+1)}}{\left(1-\alpha^{2(2 k+1)}\right)^{i}}+\sum_{i=1}^{2 n+2} D(i, h) \cdot \frac{\alpha^{2(2 k+1)(h+i)}}{\left(1-\alpha^{2(2 k+1)}\right)^{i}} \\
-\sum_{i=1}^{2 n+2} C(i, h) \cdot \frac{\beta^{2 i(2 k+1)}}{\left(1-\beta^{2(2 k+1)}\right)^{i}}-\sum_{i=1}^{2 n+2} D(i, h)
\end{gathered}
$$




$$
\begin{aligned}
& \cdot \frac{\beta^{2(2 k+1)(h+i)}}{\left(1-\beta^{2(2 k+1)}\right)^{i}}=\sum_{i=1}^{2 n+2} C(i, h) \\
& \cdot \frac{(-1)^{i} \alpha^{i(2 k+1)}-\beta^{i(2 k+1)}}{\left(\alpha^{2 k+1}-\beta^{2 k+1}\right)^{i}}+\sum_{i=1}^{2 n+2} D(i, h) \\
& \cdot \frac{(-1)^{i} \alpha^{i(2 k+1)+2 h(2 k+1)}-\beta^{i(2 k+1)+2 h(2 k+1)}}{\left(\alpha^{2 k+1}-\beta^{2 k+1}\right)^{i}} .
\end{aligned}
$$

Note that $2 U_{2 k}(x) T_{(2 i-1)(2 k+1)}(x)=U_{2 i(2 k+1)-1}(x)-$ $U_{(2 i-2)(2 k+1)-1}(x)$; from the definitions of $U_{n}(x)$ and $T_{n}(x)$ we have

$$
\begin{aligned}
& \sum_{i=1}^{2 n+2} C(i, h) \cdot \frac{(-1)^{i} \alpha^{i(2 k+1)}-\beta^{i(2 k+1)}}{\left(\alpha^{2 k+1}-\beta^{2 k+1}\right)^{i}} \\
& =\sum_{i=1}^{n+1}\left(\frac{C(2 i, h)}{\left(x^{2}-1\right)^{i-1 / 2}} \cdot \frac{2 U_{2 i(2 k+1)-1}(x)}{4^{i} U_{2 k}^{2 i}(x)}\right. \\
& \sum_{m=1}^{h}\left(T_{m}^{\prime}(x)\right)^{2 n}=\frac{(-1)^{n}}{4^{n}\left(x^{2}-1\right)^{n}}\left[\frac{(2 n) !}{(n !)^{2}} \sum_{m=1}^{h} m^{2 n}\right. \\
& \left.+2 \sum_{k=1}^{n}\left(\begin{array}{c}
2 n \\
n-k
\end{array}\right)(-1)^{k} \sum_{m=1}^{h} m^{2 n} T_{2 k m}(x)\right] .
\end{aligned}
$$$$
\left.-\frac{C(2 i-1, h)}{\left(x^{2}-1\right)^{i-1 / 2}} \cdot \frac{T_{(2 i-1)(2 k+1)}(x)}{4^{i-1} U_{2 k}^{2 i-1}(x)}\right)
$$$$
=\sum_{i=1}^{n+1} \frac{C(2 i, h)}{\left(x^{2}-1\right)^{i-1 / 2}} \cdot \frac{2 U_{2 i(2 k+1)-1}(x)}{4^{i} U_{2 k}^{2 i}(x)}
$$$$
-\sum_{i=1}^{n+1} \frac{C(2 i-1, h)}{\left(x^{2}-1\right)^{i-1 / 2}}
$$$$
\frac{2 U_{2 i(2 k+1)-1}(x)-2 U_{(2 i-2)(2 k+1)-1}(x)}{4^{i} U_{2 k}^{2 i}(x)},
$$$$
\sum_{i=1}^{2 n+2} D(i, h) \cdot \frac{(-1)^{i} \alpha^{i(2 k+1)+2 h(2 k+1)}-\beta^{i(2 k+1)+2 h(2 k+1)}}{\left(\alpha^{2 k+1}-\beta^{2 k+1}\right)^{i}}
$$$$
=\sum_{i=1}^{n+1} \frac{D(2 i, h)}{\left(x^{2}-1\right)^{i-1 / 2}} \cdot \frac{2 U_{(2 i+2 h)(2 k+1)-1}(x)}{4^{i} U_{2 k}^{2 i}(x)}
$$$$
-\sum_{i=1}^{n+1} \frac{D(2 i-1, h)}{\left(x^{2}-1\right)^{i-1 / 2}}
$$$$
\cdot \frac{2 U_{(2 i+2 h)(2 k+1)-1}(x)-2 U_{(2 i-2+2 h)(2 k+1)-1}(x)}{4^{i} U_{2 k}^{2 i}(x)} .
$$

Combining (27)-(29) we may deduce that

$$
\begin{aligned}
\sum_{m=1}^{h} & \left(m^{2 n+1} \alpha^{2 m(2 k+1)}-m^{2 n+1} \beta^{2 m(2 k+1)}\right) \\
& =\sum_{i=1}^{n+1} \frac{R(i, h) U_{2 i(2 k+1)-1}(x)+S(i, h) U_{(2 i-2)(2 k+1)-1}(x)}{4^{i}\left(x^{2}-1\right)^{i-1 / 2} U_{2 k}^{2 i}(x)} \\
& +\sum_{i=1}^{n+1} \frac{H(i, h) U_{(2 i+2 h)(2 k+1)-1}(x)+K(i, h) U_{(2 i-2+2 h)(2 k+1)-1}(x)}{4^{i}\left(x^{2}-1\right)^{i-1 / 2} U_{2 k}^{2 i}(x)} .
\end{aligned}
$$

From (26) and (30) we have the identity

$$
\begin{aligned}
\sum_{m=1}^{h} & \left(T_{2 m}^{\prime}(x)\right)^{2 n+1}=\sum_{i=1}^{n+1} \sum_{k=0}^{n}\left(\begin{array}{c}
2 n+1 \\
n-k
\end{array}\right)(-1)^{n-k} \\
\cdot & \frac{R(i, h) U_{2 i(2 k+1)-1}(x)+S(i, h) U_{(2 i-2)(2 k+1)-1}(x)}{4^{i}\left(x^{2}-1\right)^{n+i} U_{2 k}^{2 i}(x)} \\
+ & \sum_{i=1}^{n+1} \sum_{k=0}^{n}\left(\begin{array}{c}
2 n+1 \\
n-k
\end{array}\right)(-1)^{n-k} \\
& \cdot \frac{H(i, h) U_{(2 i+2 h)(2 k+1)-1}(x)+K(i, h) U_{(2 i-2+2 h)(2 k+1)-1}(x)}{4^{i}\left(x^{2}-1\right)^{n+i} U_{2 k}^{2 i}(x)} .
\end{aligned}
$$

This proves Theorem 1 .

Now we prove Theorem 2. From the first identity of

From (II) of Lemma 2 and the method of proving (30) we have

$$
\begin{aligned}
2 \sum_{m=1}^{h} & m^{2 n} T_{2 k m}(x)=\sum_{m=1}^{h} m^{2 n}\left(\alpha^{2 k m}+\beta^{2 k m}\right) \\
= & \sum_{i=1}^{2 n+1} C(i, h) \cdot \frac{\alpha^{2 k i}}{\left(1-\alpha^{2 k}\right)^{i}}+\sum_{i=1}^{2 n+1} D(i, h) \cdot \frac{\alpha^{2 k(h+i)}}{\left(1-\alpha^{2 k}\right)^{i}} \\
& +\sum_{i=1}^{2 n+1} C(i, h) \cdot \frac{\beta^{2 k i}}{\left(1-\beta^{2 k}\right)^{i}}+\sum_{i=1}^{2 n+1} D(i, h) \\
& \cdot \frac{\beta^{2 k(h+i)}}{\left(1-\beta^{2 k}\right)^{i}} \\
= & \sum_{i=1}^{n} \frac{2 C(2 i, h) T_{2 i k}(x)}{4^{i}\left(x^{2}-1\right)^{i} U_{k-1}^{2 i}(x)} \\
& -\sum_{i=1}^{n+1} \frac{2 C(2 i-1, h)\left(T_{2 i k}(x)-T_{2(i-1) k}(x)\right)}{4^{i}\left(x^{2}-1\right)^{i} U_{k-1}^{2 i}(x)} \\
& +\sum_{i=1}^{n} \frac{2 D(2 i, h) T_{2 k(h+i)}(x)}{4^{i}\left(x^{2}-1\right)^{i} U_{k-1}^{2 i}(x)} \\
& \sum_{i=1}^{n+1} \frac{2 D(2 i-1, h)\left(T_{2 k(h+i)}(x)-T_{2 k(h+i-1)}(x)\right)}{4^{i}\left(x^{2}-1\right)^{i} U_{k-1}^{2 i}(x)}
\end{aligned}
$$

Combining (32) and (33) we may immediately deduce

$$
\begin{aligned}
& \sum_{m=1}^{h}\left(T_{m}^{\prime}(x)\right)^{2 n}=\frac{(-1)^{n}}{4^{n}\left(x^{2}-1\right)^{n}} \cdot \frac{(2 n) !}{(n !)^{2}} \cdot\left(\sum_{m=1}^{h} m^{2 n}\right) \\
& \quad+\sum_{i=1}^{n+1} \sum_{k=1}^{n}\left(\begin{array}{c}
2 n \\
n-k
\end{array}\right)(-1)^{n-k}
\end{aligned}
$$




$$
\begin{aligned}
& \cdot \frac{r(i, h) T_{2 k i}(x)+s(i, h) T_{2 k(i-1)}(x)}{4^{n+i}\left(x^{2}-1\right)^{n+i} U_{k-1}^{2 i}(x)} \\
& +\sum_{i=1}^{n+1} \sum_{k=1}^{n}\left(\begin{array}{c}
2 n \\
n-k
\end{array}\right)(-1)^{n-k} \\
& \cdot \frac{p(i, h) T_{2 k(h+i)}(x)+q(i, h) T_{2 k(h+i-1)}(x)}{4^{n+i}\left(x^{2}-1\right)^{n+i} U_{k-1}^{2 i}(x)} .
\end{aligned}
$$

This proves Theorem 2 .

Now we prove Corollary 3. Applying the second formula of Lemma 1 with $n=0$ and formula (A) of Lemma 2 we have

$$
\begin{aligned}
& \sum_{m=1}^{h} T_{2 m}^{\prime}(x)=\sum_{m=1}^{h} 2 m U_{2 m-1}(x)=\frac{1}{\sqrt{x^{2}-1}} \\
& \cdot \sum_{m=1}^{h} m\left(\alpha^{2 m}-\beta^{2 m}\right) \\
& =\frac{1}{\sqrt{x^{2}-1}}\left[\frac{\alpha^{2}-(h+1) \alpha^{2 h+2}+h \alpha^{2 h+4}}{\left(1-\alpha^{2}\right)^{2}}\right. \\
& \left.-\frac{\beta^{2}-(h+1) \beta^{2 h+2}+h \beta^{2 h+4}}{\left(1-\beta^{2}\right)^{2}}\right] \\
& =\frac{h U_{2 h+1}(x)-(h+1) U_{2 h-1}(x)}{2\left(x^{2}-1\right)} .
\end{aligned}
$$

This proves Corollary 3.

Similarly, applying the first formula of Lemma 1 with $n=$ 1 and formula (B) of Lemma 2 we have

$$
\begin{aligned}
& \sum_{m=1}^{h}\left(T_{m}^{\prime}(x)\right)^{2}=\frac{1}{4\left(x^{2}-1\right)}\left[-2 \sum_{m=1}^{h} m^{2}\right. \\
& \left.\quad+2 \sum_{m=1}^{h} m^{2} T_{2 m}(x)\right] \\
& \quad=\frac{1}{4\left(x^{2}-1\right)}\left[-\frac{h(h+1)(2 h+1)}{3}\right. \\
& \left.\quad+\sum_{m=1}^{h} m^{2}\left(\alpha^{2 m}+\beta^{2 m}\right)\right] .
\end{aligned}
$$

Note that

$$
\begin{aligned}
\sum_{m=1}^{h} m^{2}\left(\alpha^{2 m}+\beta^{2 m}\right) \\
=\frac{\alpha^{2}+\alpha^{4}}{\left(1-\alpha^{2}\right)^{3}}-\frac{(h+1)^{2} \alpha^{2 h+2}}{1-\alpha^{2}}-\frac{(2 h+3) \alpha^{2 h+4}}{\left(1-\alpha^{2}\right)^{2}} \\
\quad-\frac{2 \alpha^{2 h+6}}{\left(1-\alpha^{2}\right)^{3}}+\frac{\beta^{2}+\beta^{4}}{\left(1-\beta^{2}\right)^{3}}-\frac{(h+1)^{2} \beta^{2 h+2}}{1-\beta^{2}}
\end{aligned}
$$

$$
\begin{aligned}
& -\frac{(2 h+3) \beta^{2 h+4}}{\left(1-\beta^{2}\right)^{2}}-\frac{2 \beta^{2 h+6}}{\left(1-\beta^{2}\right)^{3}} \\
= & \frac{(h+1)^{2}\left(\alpha^{2 h+4}-\alpha^{2 h+2}\right)-(2 h+3) \alpha^{2 h+4}}{\left(1-\alpha^{2}\right)^{2}} \\
& +\frac{2\left(\alpha^{2 h+8}-\alpha^{2 h+6}\right)}{\left(1-\alpha^{2}\right)^{4}} \\
& +\frac{(h+1)^{2}\left(\beta^{2 h+4}-\beta^{2 h+2}\right)-(2 h+3) \beta^{2 h+4}}{\left(1-\beta^{2}\right)^{2}} \\
& +\frac{2\left(\beta^{2 h+8}-\beta^{2 h+6}\right)}{\left(1-\beta^{2}\right)^{4}} \\
= & \frac{\left(h^{2}-2\right) T_{2 h+2}(x)-(h+1)^{2} T_{2 h}(x)}{2\left(x^{2}-1\right)} \\
+ & \frac{T_{2 h+4}(x)-T_{2 h+2}(x)}{4\left(x^{2}-1\right)^{2}} .
\end{aligned}
$$

Combining (36) and (37) we have the identity

$$
\begin{aligned}
\sum_{m=1}^{h}\left(T_{m}^{\prime}(x)\right)^{2} & \\
= & -\frac{h(h+1)(2 h+1)}{12\left(x^{2}-1\right)} \\
& +\frac{\left(h^{2}-2\right) T_{2 h+2}(x)-(h+1)^{2} T_{2 h}(x)}{8\left(x^{2}-1\right)^{2}} \\
& +\frac{T_{2 h+4}(x)-T_{2 h+2}(x)}{16\left(x^{2}-1\right)^{3}} .
\end{aligned}
$$

This completes the proof of all results.

Some Comments. In our theorems, we can give the exact expressions for all constants $R(i, h), S(i, h), r(i, h)$, and $s(i, h)$, if $k$ is a small positive integer. If $k$ is large enough, then we can only give an exact computational method for these constants (because of the reason of Lemma 2), but the computation is more complex, and so we have not obtained the exact expression for $R(i, h), S(i, h), r(i, h)$, and $s(i, h), 1 \leq i \leq h$.

\section{Conflict of Interests}

The authors declare that there is no conflict of interests regarding the publication of this paper.

\section{Acknowledgments}

The authors would like to thank the referee for his very helpful and detailed comments, which have significantly improved 
the presentation of this paper. This work is supported by the P. S. F. (2014JQ2-1005) and N. S. F. (11371291) of China.

\section{References}

[1] R. Ma and W. Zhang, "Several identities involving the Fibonacci numbers and Lucas numbers," The Fibonacci Quarterly, vol. 45, no. 2, pp. 164-170, 2007.

[2] X. Wang and D. Han, "Some identities related to Dedekind sums and the Chebyshev polynomials," International Journal of Applied Mathematics \& Statistics, vol. 51, no. 21, pp. 334-339, 2013.

[3] C. Cesarano, "Identities and generating functions on Chebyshev polynomials," Georgian Mathematical Journal, vol. 19, no. 3, pp. 427-440, 2012.

[4] C.-L. Lee and K. B. Wong, "On Chebyshev's polynomials and certain combinatorial identities," Bulletin of the Malaysian Mathematical Sciences Society, vol. 34, no. 2, pp. 279-286, 2011.

[5] E. H. Doha, A. H. Bhrawy, and S. S. Ezz-Eldien, "Numerical approximations for fractional diffusion equations via a Chebyshev spectral-tau method," Central European Journal of Physics, vol. 11, no. 10, pp. 1494-1503, 2013.

[6] A. H. Bhrawy and M. A. Zaky, "A method based on the Jacobi tau approximation for solving multi-term time-space fractional partial differential equations," Journal of Computational Physics, vol. 281, pp. 876-895, 2015.

[7] E. H. Doha, A. H. Bhrawy, R. M. Hafez, and M. A. Abdelkawy, "A Chebyshev-Gauss-Radau scheme for nonlinear hyperbolic system of first order," Applied Mathematics and Information Sciences, vol. 8, no. 2, pp. 535-544, 2014.

[8] E. H. Doha and A. H. Bhrawy, "A Jacobi spectral Galerkin method for the integrated forms of fourth-order elliptic differential equations," Numerical Methods for Partial Differential Equations, vol. 25, no. 3, pp. 712-739, 2009.

[9] A. H. Bhrawy, "An efficient Jacobi pseudospectral approximation for nonlinear complex generalized Zakharov system," Applied Mathematics and Computation, vol. 247, pp. 30-46, 2014.

[10] A. H. Bhrawy and M. A. Zaky, "Numerical simulation for twodimensional variable-order fractional nonlinear cable equation," Nonlinear Dynamics, vol. 80, no. 1-2, pp. 101-116, 2015.

[11] X. Li, "Some identities involving Chebyshev polynomials," Mathematical Problems in Engineering, vol. 2015, Article ID 950695, 5 pages, 2015. 


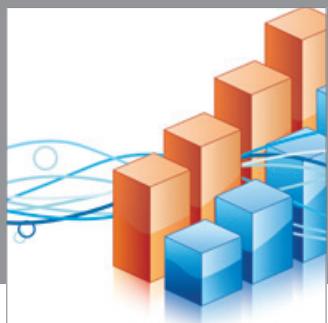

Advances in

Operations Research

mansans

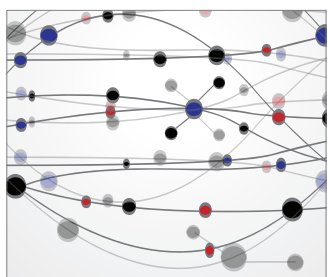

The Scientific World Journal
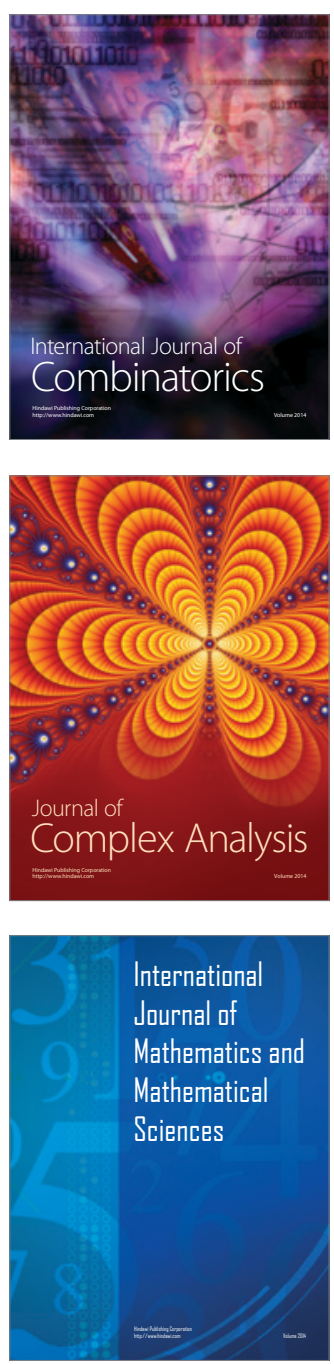
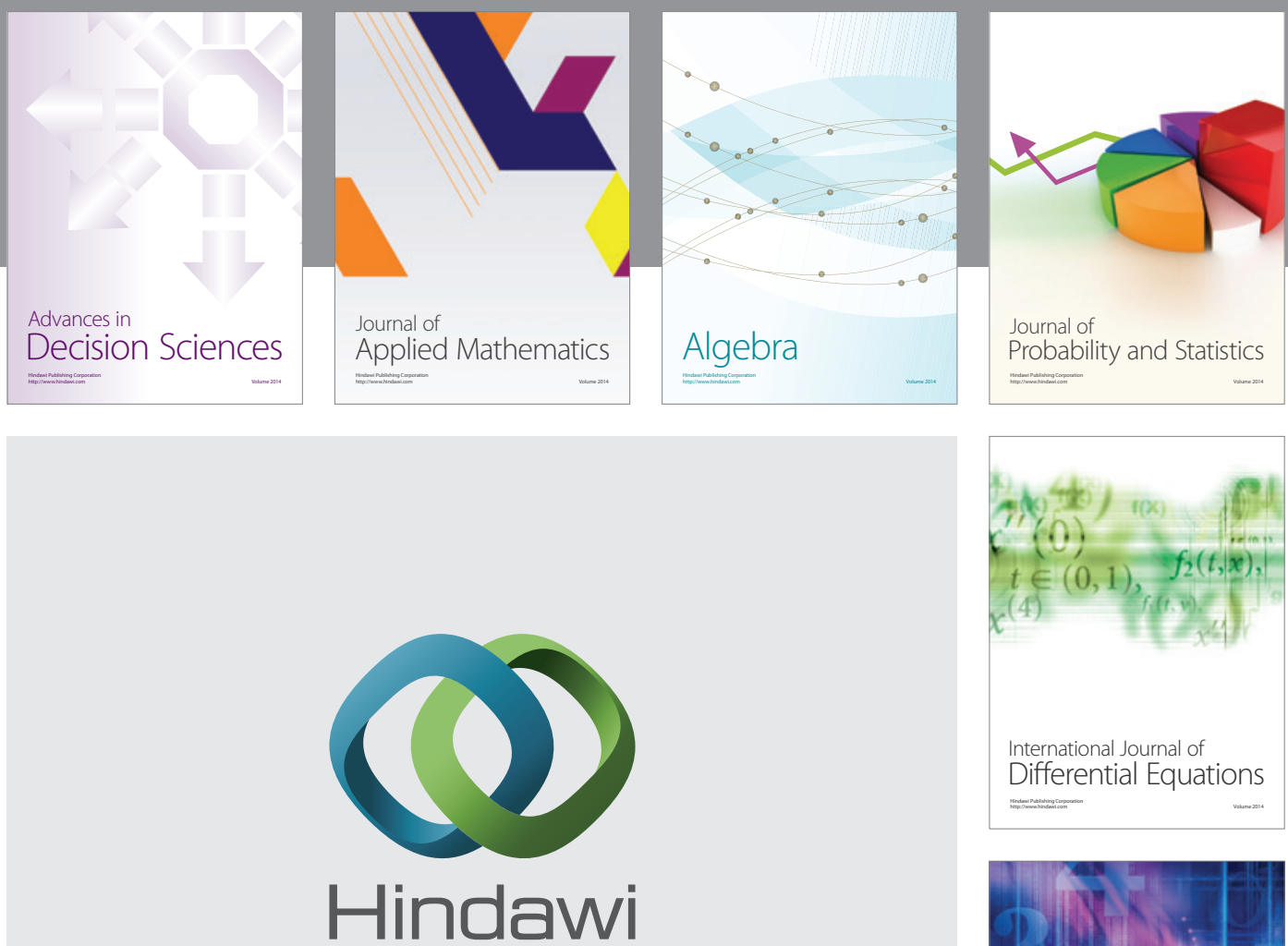

Submit your manuscripts at http://www.hindawi.com
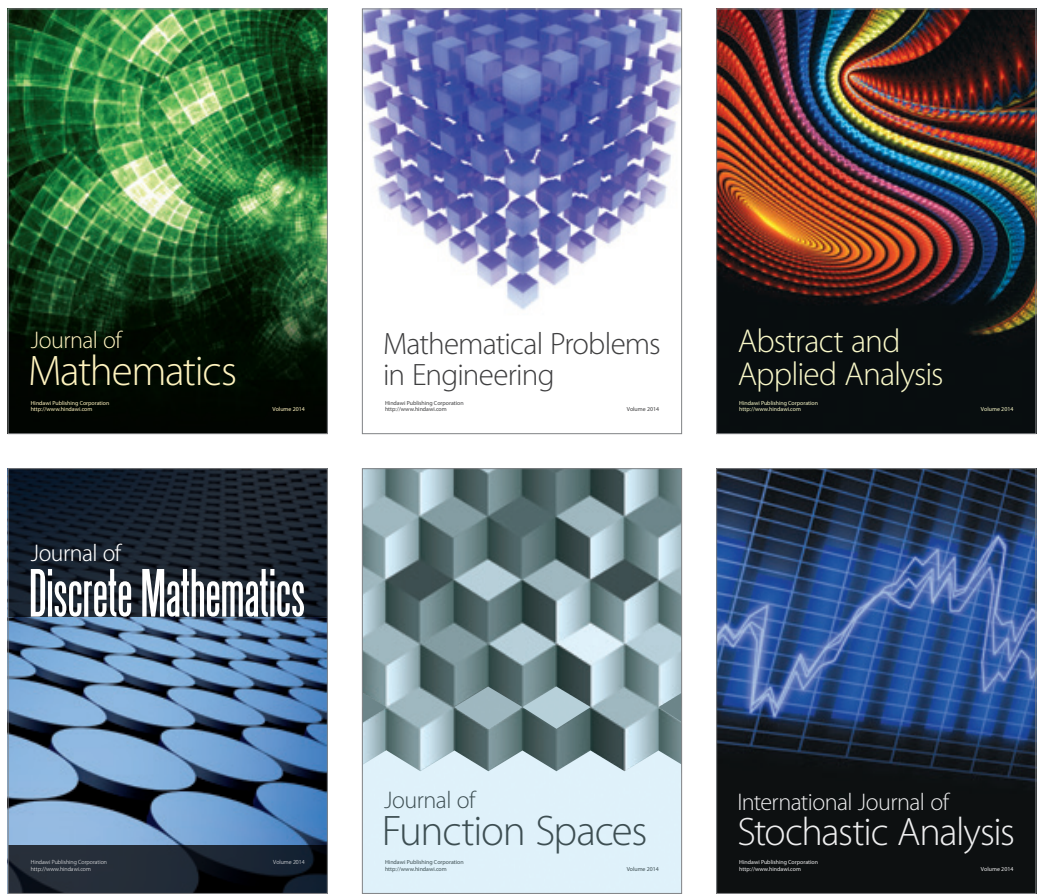

Journal of

Function Spaces

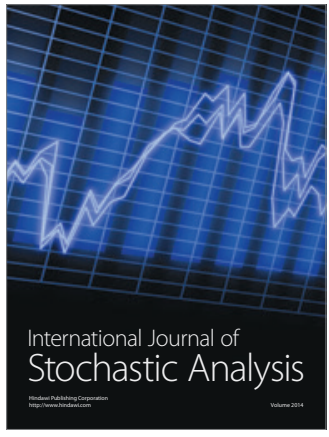

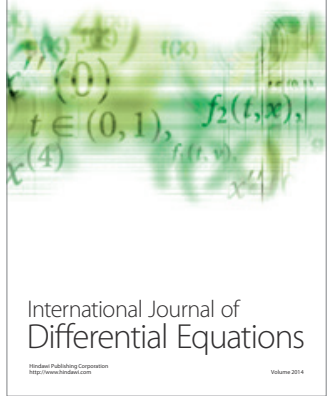
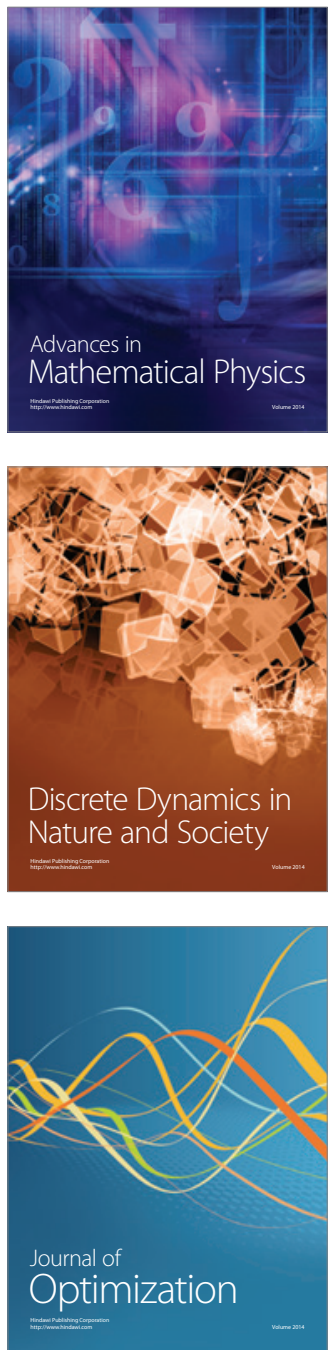